\title{
Protective effect of luteolin on skin ischemia-reperfusion injury through an AKT-dependent mechanism
}

\author{
GANG CHEN $^{1,2}$, HUGANG SHEN ${ }^{2}$, LINLIN ZANG $^{3}$, ZHONGLAN SU $^{4}$, \\ JILONG HUANG $^{2}$, YONG SUN ${ }^{1}$ and HONGWEI WANG ${ }^{1,5}$
}

\begin{abstract}
${ }^{1}$ Key Laboratory of Acupuncture and Medicine Research of Ministry of Education, Nanjing University of Chinese Medicine, Nanjing, Jiangsu 210023; ${ }^{2}$ Department of Esthetic Plastic Surgery, The First Affiliated Hospital of Nanjing University of TCM, Nanjing, Jiangsu 210029; ${ }^{3}$ Medical Laboratory, Qingdao HaiCi Medical Group, Qingdao, Shandong 266000; ${ }^{4}$ Department of Dermatology, The First Affiliated Hospital of Nanjing Medical University, Nanjing, Jiangsu 210029; ${ }^{5}$ Center for Translational Medicine and Jiangsu Key Laboratory of Molecular Medicine, Medical School of Nanjing University, Nanjing, Jiangsu 210093, P.R. China
\end{abstract}

Received April 13,2018; Accepted September 4, 2018

DOI: $10.3892 /$ ijmm.2018.3915

\begin{abstract}
Cutaneous ischemia-reperfusion (I/R) injury is one of the most crucial problems in flap surgery, which affects the survival of the skin flap and patient prognosis, luteolin, a plant derived flavonoid, has previously been shown to exert a variety of beneficial effects for reducing I/R injury in several organs. The aim of the present study was to evaluate the anti-inflammatory and anti-oxidative stress effects of luteolin on cutaneous I/R injury. The in vitro study were performed using a permanent human immortalized epidermal keratinocyte cell line ( $\mathrm{HaCaT})$, cells were cultured in the presence of luteolin and were then treated with hydrogen peroxide, the cell viability, mitochondrial membrane potential and the cell survival/apoptosis related signaling pathway activation were assessed to investigate the cytoprotective effects of luteolin. For in vivo experiments, skin flap I/R injury animal model was established in Sprague-Dawley rats, by measuring the area of flap survival, analyzing the expression of pro-inflammatory cytokine and evaluation of the histological changes in the skin tissue, the protective effects of luteolin on skin I/R injury were investigated. The function of protein kinase B (AKT) and heme oxygenase-1 (HO-1) activation on luteolin medi-
\end{abstract}

Correspondence to: Professor Hongwei Wang, Center for Translational Medicine and Jiangsu Key Laboratory of Molecular Medicine, Medical School of Nanjing University, 22 Hankou Road, Nanjing, Jiangsu 210093, P.R. China

E-mail: hwang@nju.edu.cn

Mr. Yong Sun, Key Laboratory of Acupuncture and Medicine Research of Ministry of Education, Nanjing University of Chinese Medicine, 138 Xianlin Road, Nanjing, Jiangsu 210023, P.R. China

E-mail: suny7635@163.com

Key words: luteolin, skin flap surgery, ischemia-reperfusion injury, reactive oxygen species ated I/R injury protection was assessed by administration of phosphoinositide-3-kinase/AKT inhibitor LY294002 and HO-1 inhibitor ZNPP. The results showed that luteolin treatment significantly increased the viability of $\mathrm{HaCaT}$ cells upon exposure to hydrogen peroxide, and the administration of luteolin in vivo significantly improved skin flap survival in the I/R injury rat model. The mechanisms underlying these beneficial effects included increased phosphoinositide-3-kinase/protein kinase $\mathrm{B}$ activation, improved expression of antioxidant enzyme, and scavenging the cytotoxic effects of reactive oxygen species (ROS). Taken together, the results suggested that luteolin preconditioning yielded significant protection against cutaneous $\mathrm{I} / \mathrm{R}$ injury by protecting skin keratinocytes from ROS-induced damage.

\section{Introduction}

Skin flap surgery has been increasingly used in plastic and reconstructive surgery of a number of skin defects, and flap necrosis is the least common but most serious problem following reconstructive flap surgery. Ischemia-reperfusion (I/R) injury is a leading cause of surgical skin flap compromise and organ dysfunction. I/R injury occurs when the circulation is abruptly restored following prolonged ischemia, and the mechanisms underlying I/R injury are complex. Evidence shows that high levels of calcium and tissue neutrophil accumulation cause cellular damage (1), the generation of high level of reactive oxygen species (ROS) during reperfusion, and the induction of marked epithelial apoptosis are critical in the pathogenesis of various types of I/R injury in tissue damage and organ dysfunction (2). As skin flaps are vulnerable to surgical skin flap-induced I/R injury, reducing I/R injury in the necrotizing flaps has long been a clinical challenge.

Luteolin (3',4',5,7-tetrahydroxyflavone) is a naturally occurring polyphenol flavonoid found in several vegetables, fruits, and tea. It has been reported to exert varied pharmacological activities, including antioxidant, antimutagenic, anti-inflammatory, anti-allergic and antihypertensive activities (3). The 
beneficial effects of luteolin have been reported in various pathological conditions, including lipopolysaccharide-induced acute lung injury, endotoxin-induced uveitis (4), intestinal inflammation (5), acetaminophen-induced hepatotoxicity and asthma (6,7).

Luteolin has also been found to exert protective effects during the process of I/R injury; it has been demonstrated to protect by the alleviation of myocardial, kidney and intestinal I/R injury in animal models or clinical studies (8-10). However, whether luteolin can be used for the treatment of the cutaneous $\mathrm{I} / \mathrm{R}$ injury in skin flap surgery is of interest and warrants investigation. Therefore, the present study aimed to evaluate the anti-ischemic effect of luteolin in skin flap surgery and investigate its potential mechanism.

\section{Materials and methods}

Reagents and antibodies. Luteolin was purified and provided by the Key Laboratory of Bioactive Substances and Resources Utilization of Chinese Herbal Medicine, Ministry of Education (Beijing, China) and the purity of the product was $>98 \%$, detected by HPLC (UV). The molecular structure is shown in Fig. 1A. Monoclonal antibodies against protein kinase B (AKT; no. 4691), phosphor-AKT (Ser473; no. 4060) and horseradish peroxidase-conjugated anti-mouse or anti Rabbit IgG (nos. 7076 and 7074) were purchased from Cell Signaling Technology, Inc. (Boston, MA, USA). Monoclonal antibodies against heme oxygenase-1 (HO-1; no. ab13243), B-cell lymphoma 2 (BCL-2; no. ab692), BCL-2-associated X protein (BAX; no. ab32503), activated caspase-3 (no. ab2302) and GAPDH (no. ab9484) were purchased from Abcam (Cambridge, MA, USA). Alexa 488-conjugated goat anti-rabbit secondary antibodies (no. A-11078) were purchased from Thermo Fisher Scientific, Inc. (Waltham, MA, USA). The Pierce BCA Protein Assay kit was purchased from Thermo Fisher Scientific, Inc.

Cell culture. Cultured immortalized HaCaT human keratinocyte cells were obtained from Nanjing KeyGen Biotech Co., Ltd. (Nanjing, China) (11). The cells were grown in DMEM (Thermo Fisher Scientific, Inc.) supplemented with $10 \%$ fetal bovine serum (FBS; Thermo Fisher Scientific, Inc.), $100 \mathrm{U} / \mathrm{ml}$ penicillin, and $100 \mu \mathrm{g} / \mathrm{ml}$ streptomycin, cultured at $37^{\circ} \mathrm{C}$ in $5 \%$ $\mathrm{CO}_{2}$. When $80 \%$ confluence was achieved, the HaCaT cells were treated with hydrogen peroxide $(100 \mu \mathrm{M})$ for $2 \mathrm{~h}$ with or without the pretreatment of indicated concentrations $(3,6,12$, $25 \mu \mathrm{g} / \mathrm{ml}$ ) of luteolin for $12 \mathrm{~h}$ at $37^{\circ} \mathrm{C}$ in $5 \% \mathrm{CO}_{2}$.

Cell viability and apoptosis assay. Cell viability was assessed using standard MTS methods. Briefly, the cells were seeded in triplicate at a density of $1 \times 10^{5}$ cells $/ \mathrm{ml}$ in a 96 -well plate, and cell viability assays were performed using the CellTiter 96 Aqueous One Solution Cell Proliferation Assay kit (Promega Corporation, Madison, WI, USA), the absorbance at $490 \mathrm{~nm}$ was measured using a microplate reader (BioTek Instruments, Inc., Winooski, VT, USA).

For the apoptosis assays, a fluorescein-conjugated Annexin V (Annexin V-FITC) staining assay kit (BD Biosciences, San Jose, CA, USA) was used to quantitatively assess the level of induced cell apoptosis. Briefly, the cells were washed with PBS and stained with $5 \mu \mathrm{l}$ of Annexin V-FITC and $5 \mu \mathrm{l}$ of propidium iodide (PI) in each sample to quantify the cell number at different stages of cell death. Following incubation at room temperature in the dark for $15 \mathrm{~min}$, the percentages of apoptotic cells were quantified as a percentage of the Annexin V-positive population with a FACSCalibur flow cytometer (BD Biosciences), and the data were analyzed with FlowJo Version 7.6.2 software (Tree Star, Inc., Ashland, OR, USA).

Measurement of mitochondrial membrane potential (MMP). The MMP values were determined using the dual-emission potential-sensitive probe 6,6'-tetrachloro-1,1',3,3'-tetraethylimidacarbocyanineiodide (JC-1; Sigma-Aldrich; Merck KGaA, Darmstadt, Germany). Briefly, the cells were seeded in the camber slides at a density of $5 \times 10^{5}$ cells/well in $200 \mu \mathrm{l}$ culture medium and incubated with $10 \mu \mathrm{M}$ of JC-1 for $20 \mathrm{~min}$ at $37^{\circ} \mathrm{C}$ in the dark. The JC-1 was then removed, and the cells were washed with cold PBS to remove unbound dye. The quantity of JC-1 retained in the cells was assessed with a laser scanning confocal microscope system (Olympus Corporation, Tokyo, Japan).

Western blot analysis. Skin keratinocytes were incubated for a period of time following hydrogen peroxide exposure, following which cell lysates were collected by lysis in RIPA buffer, and the concentration of protein was detected using a Pierce BCA protein kit purchased from Thermo Fisher Scientific, Inc. Equal quantities of proteins $(20 \mu \mathrm{g})$ were mixed with loading buffer and subjected to electrophoresis using 10\% SDS-polyacrylamide gels. The separated proteins were transferred onto polyvinylidene fluoride membranes and non-specific bindings were blocked with $5 \%$ (w/v) skim milk dissolved in tris-buffered saline with Tween. The membranes were then subjected to immunoblot analysis with the appropriate antibodies $(1 ; 1,000$ dilution for the primary antibodies for $2 \mathrm{~h}$ at room temperature; 1:2,000 dilution for the secondary antibodies for $1 \mathrm{~h}$ at room temperature). The immune-reactive protein bands were visualized using an enhanced chemiluminescence detection system (GE Healthcare Life Sciences, Chalfont, UK) followed by autoradiography. A G:Box Bioimaging system (Syngene, Frederick, MD, USA) was used to assess autoradiographic signals and bands were quantified using GeneTools Image Analysis Software version 4.3.7 (Syngene).

Animal preparation and experimental groups. The animal experiments were performed in the Experimental Animal Laboratory of Nanjing University School of Medicine (Nanjing, China), and approved by the Institutional Animal Care and Use Committee of Nanjing University. A total of 18 male 8-10 weeks-old Sprague-Dawley rats weighing 220-280 g were purchased from Research Institute of Model Organisms at Nanjing University, the rats were housed in separate cages at $25^{\circ} \mathrm{C}$ and in a 12-h light/dark lighting system. All animals has free access to food and water. The rats were randomly allocated into three groups: Mock control group (Ctl), I/R injury group (I/R), and I/R injury with luteolin treatment group (I/R + Luo).

Development of the ischemic flap rat model. The rats were anesthetized with an intraperitoneal (i.p.) injection of 
A

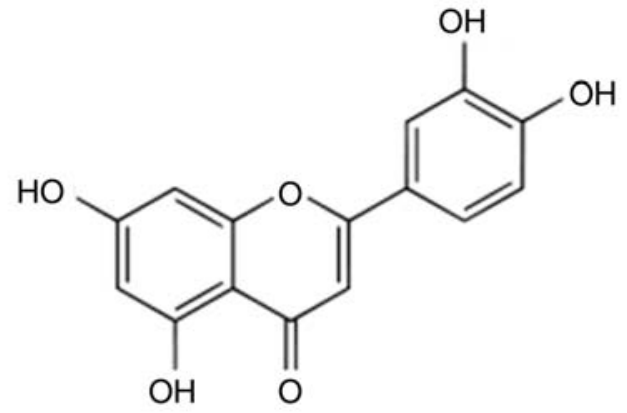

B

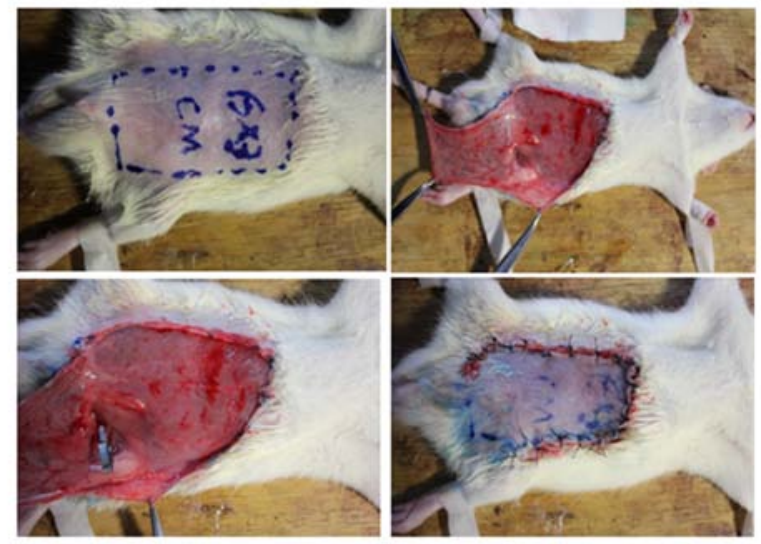

Figure 1. Chemical structure of the luteolin and the surgical procedure (A) Cell viability of $\mathrm{HaCaT}$ cells were detected using an MTS assay (B) Diagram showing the surgical procedure. Briefly, the rat was anesthetized and an island skin flap measuring $3 \times 6 \mathrm{~cm}$ over the lower chest and abdomen was raised; the flaps were transected proximally, leaving the superficial epigastric vessels as the only connection, and epigastric vessels close to the femoral artery and vein were occluded with a 2-V microvascular clamp during the ischemic period. The clamps were removed following $4 \mathrm{~h}$ of ischemia.

ketamine $(100 \mathrm{mg} / \mathrm{ml})$ and xylazine $(20 \mathrm{mg} / \mathrm{ml})$ at a total dose of $0.2 \mathrm{ml} / 100 \mathrm{~g}$ of body weight. Abdominal hair was removed with an electric clipper, and all surgical procedures were performed under sterile conditions. The borders of the flaps were outlined on the abdomen using a template measuring $3 \times 6 \mathrm{~cm}$. The flap was raised with the base at the left inferior epigastric artery, including the skin and the intimately attached panniculus carnosus, as previously described $(11,12)$. Ischemia was induced by applying a single microvascular clamp across the femoral vascular pedicle, and the flap was sutured to the donor bed using a 4/0 polypropylene suture. Following $4 \mathrm{~h}$ of ischemia, the clamps were removed. The rats in the mock control group received the flap elevation and vessel dissection but no ischemic insult. The surgical procedure is shown in Fig 1B. To assess the functions of phosphoinositide-3-kinase (PI3K)/AKT and HO-1 in luteolin-mediated tissue protection, HO-1 inhibitor (ZnPP) or PI3K/AKT inhibitor (LY294002) was administrated to the rats on day 1 and day 4 post-I/R injury at doses of 3 and $100 \mathrm{mg} / \mathrm{kg}$ body weight (i.p.). The surviving area of the skin flap was then measured.

Assessment of skin flap survival. Following surgery, all animals were sacrificed on post-operative day 7. Gross observation was performed to identify the line of demarcation between the viable and the necrotic tissue. The dorsum of the rat in each group was covered with clear paper in anesthesia to accurately measure the surviving or necrotic areas of the flap, and the flap was cut into two sections: Viable and necrotic. The entire flap and the necrotic and viable regions were measured using two-dimensional planimetry in a blinded-manner. The surviving proportions of the flaps were determined as a percentage of the entire flap area (surviving flap proportion=viable flap area/total area x100\%). Following assessment, the rats were sacrificed with an overdose of sodium pentothal.

Reverse transcription-quantitative polymerase chain reaction (RT-qPCR) analysis. The transcriptional expression of interleukin (IL)-1 $\beta$, tumor necrosis factor (TNF)- $\alpha$ and IL-6 were detected by RT-qPCR analysis. Total RNA was extracted from the skin flap tissue on day 1 post-I/R using an RNAeasy Micro kit (Qiagen GmbH, Hilden, Germany) according to the manufacturer's protocol. mRNA was reverse transcribed into cDNA with PrimeScript RT Master mix (Takara Bio, Inc., Otsu, Japan). SYBR green qPCR was performed using PCR Master mix (Thermo Fisher Scientific, Inc.). Each cDNA reaction was prepared from $1 \mu \mathrm{g}$ RNA, diluted to $100 \mu \mathrm{l}$ of the final volume and $1 \mu \mathrm{l}$ cDNA was subsequently used for each PCR reaction, and the reaction mixture had a total volume of $20 \mu \mathrm{l}$ containing $10 \mu \mathrm{l}$ PCR Master Mix (2X), $0.5 \mu \mathrm{l} \mathrm{PCR}$ forward primer $(10 \mathrm{mM}), 0.5 \mu \mathrm{l}$ PCR reverse primer $(10 \mathrm{mM})$ and $8 \mu \mathrm{l} \mathrm{H}_{2} \mathrm{O}$. The PCR conditions were as follows: $95^{\circ} \mathrm{C}$ for $30 \mathrm{sec}$ for pre-incubation, $95^{\circ} \mathrm{C}$ for $5 \mathrm{sec}$ and $60^{\circ} \mathrm{C}$ for $30 \mathrm{sec}$ for amplification; $95^{\circ} \mathrm{C}$ for $10 \mathrm{sec}$ and $65^{\circ} \mathrm{C}$ for $10 \mathrm{sec}$ to melting curve, and $40^{\circ} \mathrm{C}$ for $30 \mathrm{sec}$ for cooling. The following primer pairs were used: IL-1 $\beta, 5^{\prime}$-GGAACCCGTGTCTTC CTAAAG-3' (forward) and 5'-CTGACTTGGCAGAGG ACAAAG-3' (reverse); TNF- $\alpha, 5$ '-CCAACAAGGAGGAGA AGTTCC-3' (forward) and 5'-CTCTGCTTGGTGGTTTGC TAC-3' (reverse); IL-6, 5'-GAAAGTCAACTCCATCTGCC-3' (forward) and 5'-CATAGCACACTACGTTTGCC-3' (reverse); first measure-actin, 5'-AACCCTAAGGCCAACCGTGAA AAG-3' (forward) and 5'-TCATGAGGTAGTCTGTCAGGT-3' (reverse).

The relative expression of target genes was determined to $\beta$-actin and was calculated using the $2^{-\Delta \Delta \mathrm{Cq}}$ method. The relative mRNA expression was quantified as described previously (13).

Assessment of oxidative stress status. The oxidative stress status of the flaps was assessed by measuring the superoxide dismutase (SOD) activity and the content of myeloperoxidase (MPO) and malondialdehyde (MDA) in the skin flap tissue. Tissue samples $(1 \times 1 \mathrm{~cm})$ were separated from the central area of the surgical flaps in each group; these samples were weighed, homogenized, and diluted to $10 \%(\mathrm{v} / \mathrm{v})$ in an ice bath. The homogenate was then centrifuged at $600 \mathrm{x} \mathrm{g}$ for $15 \mathrm{~min}$ at $4^{\circ} \mathrm{C}$ and the supernatant solution was collected. The activity of SOD and the levels of MPO and MDA in the homogenate were then determined using a commercial kit following the protocol suggested by the manufacturer (Nanjing Jiancheng Bioengineering Institute, Nanjing, China).

Immunofluorescent staining. Tissue specimens were embedded in paraffin following fixation in $10 \%$ formalin. The sections (4-6 $\mu \mathrm{m})$ were deparaffinized in xylene and 

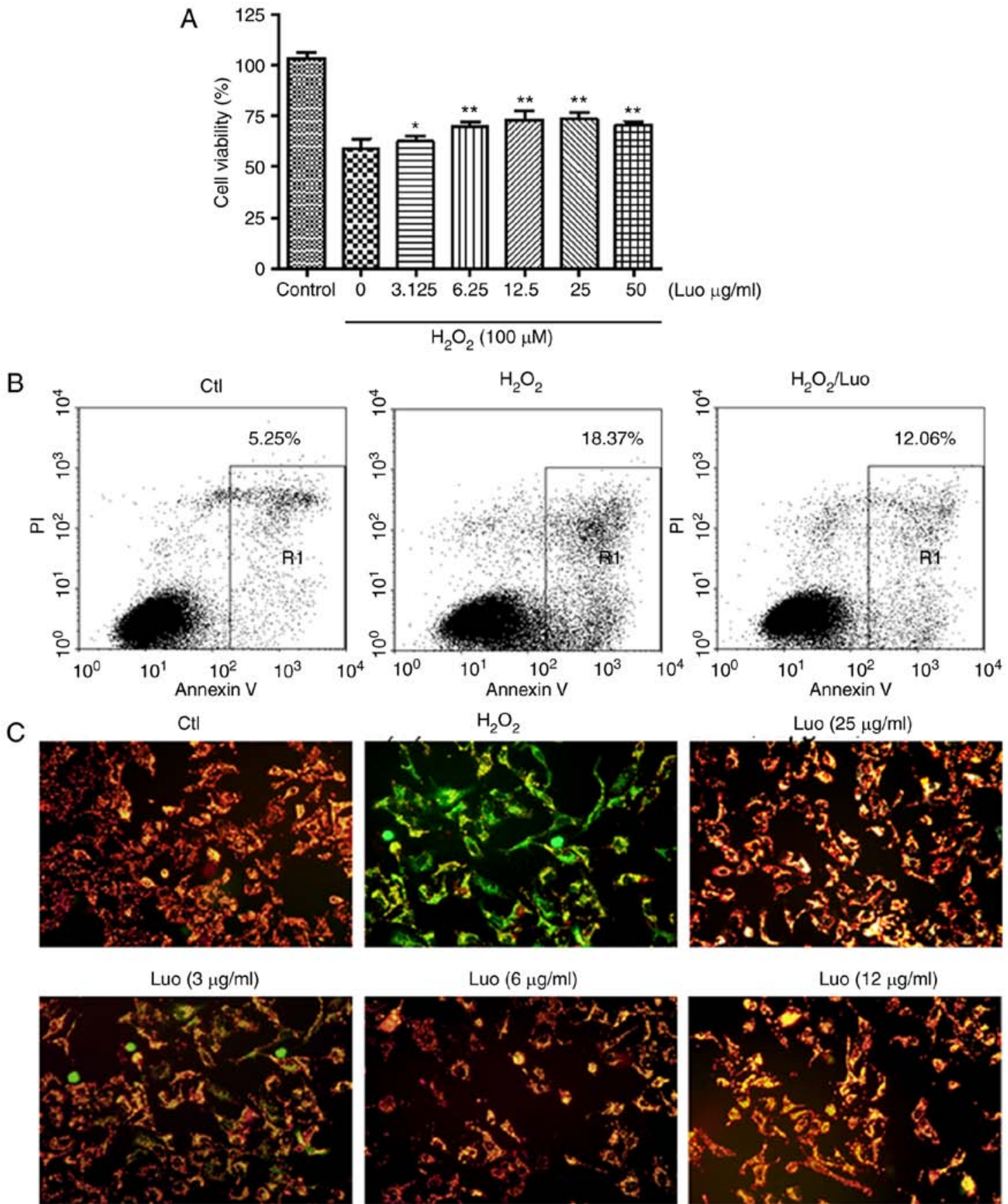

Figure 2. Luteolin preconditioning decreases the cytotoxic effect of hydrogen peroxide in HaCaT keratinocyte cells. (A) Cell viability of HaCaT cells detected using a MTT assay in the presence of hydrogen peroxide and luteolin pretreatment; (B) Percentage of apoptotic cells was assessed by FACS with Annexin-V and PI staining. (C) Mitochondrial membrane potential assay was performed using 6,6'-tetrachloro-1,1',3,3'-tetraethylimidacarbocyanineiodide staining (magnification, $\mathrm{x} 100)$. Results are expressed as the mean \pm standard deviation ( $\mathrm{P}<0.05,{ }^{* * *} \mathrm{P}<0.01$ compared with the $\mathrm{H}_{2} \mathrm{O}_{2}$-treated group). Ctl, control; Luo, luteolin; PI, propidium iodide.

hydrated with ethanol. The sections were incubated with $1 \%$ bovine serum albumin (Sigma-Aldrich; Merck KGaA) in PBS/Tween to block unspecific binding of the antibodies. Following overnight incubation at $4^{\circ} \mathrm{C}$, the sections were incubated with primary antibodies (1:500 for P-AKT, HO-1 and active Caspase-3) for $2 \mathrm{~h}$ followed by the secondary antibodies $(1: 1,000)$ for $30 \mathrm{~min}$ in a dark room at $25^{\circ} \mathrm{C}$. Nuclei were stained using DAPI solution (Molecular Probes, Thermo Fisher Scientific, Inc.). Fluorescent images were captured and visualized using an Olympus fluorescence microscope (Olympus Corporation).
Statistical analysis. Statistical analyses were performed using GraphPad Prism 5.0 (GraphPad Software, Inc., La Jolla, CA, USA). One-way analysis of variance or Student's t-test was used to test for significant difference. $\mathrm{P}<0.05$ was considered to indicate a statistically significant difference.

\section{Results}

Luteolin treatment protects human skin keratinocytes from hydrogen peroxide-induced cytotoxicity. Prior to investigating the protective properties of luteolin against hydrogen 

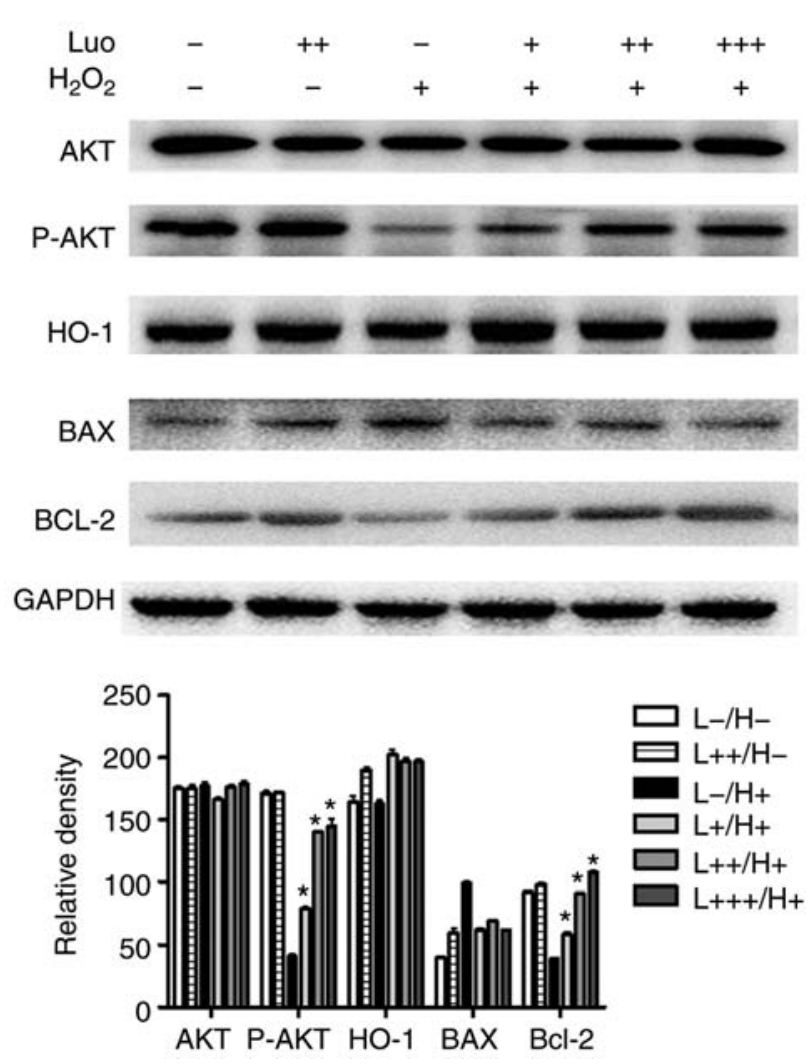

Figure 3. Effect of luteolin on hydrogen peroxide-induced keratinocyte apoptosis. The protein levels of markers of apoptosis, BAX, caspase 3 and BCL-2, were evaluated using a western blot assay. The $\mathrm{HaCaT}$ cells were exposed to $100 \mu \mathrm{M}$ of hydrogen peroxide in the presence or absence of increased concentration of luteolin.,$+ 3 \mu \mathrm{g} / \mathrm{ml} ;++, 6 \mu \mathrm{g} / \mathrm{ml} ;+++, 12 \mu \mathrm{g} / \mathrm{ml}$; AKT, protein kinase B; P-AKT, phosphorylated AKT; HO-1, heme oxygenase-1; BCL-2, B-cell lymphoma 2; BAX, BCL-2-assocated X protein; I/R, Luo, luteolin. ${ }^{*} \mathrm{P}<0.05$, vs $\mathrm{H}_{2} \mathrm{O}_{2}$ treatment.

peroxide-induced cell death, the cytotoxic effects of hydrogen peroxide were first examined in the HaCaT cells. The MTT assay indicated that the treatments with various doses of hydrogen peroxide resulted in cytotoxic effects, and cell viability was significantly decreased at a concentration of $100 \mu \mathrm{M}$. Therefore, $100 \mu \mathrm{M}$ of hydrogen peroxide was selected as the optimum concentration for the subsequent in vitro assay. To measure the protective effect of luteolin, the HaCaT cells were sham-exposed or received treatment with various doses of luteolin. MTT assays revealed that the hydrogen peroxide-induced reduction of cell viability was effectively prevented by pretreatment with luteolin (Fig. 2A).

To further evaluate the effect of luteolin on hydrogen peroxide-induced apoptosis, the HaCaT cells were stained with Annexin V and PI, and cell apoptosis was measured by FACS, as shown in Fig. 2B. Cells with a high expression of Annexin V and not expressing PI were considered early apoptotic cells, whereas cells with a high expression of Annexin V and expressing PI were classified as late apoptotic cells. The results showed that $\mathrm{HaCaT}$ cells exposed to hydrogen peroxide treatment had a high level of cell apoptosis, and treatment of these cells with luteolin significantly reduced cell apoptosis; the percentage of apoptotic cells was $12.06 \pm 2.32 \%$ (luteolin treatment group), vs. $18.37 \pm 1.92 \%$ (untreated group). This suggested that luteolin may inhibit hydrogen peroxide-induced keratinocyte apoptosis.
A change in MMP was examined as mitochondria are major sites of oxidative phosphorylation and ROS production, and they are involved in the initiation of apoptosis through membrane permeabilization (14). As shown in Fig. 2C, compared with the control group, the hydrogen peroxide-treated cells exhibited a loss of MMP; increased fluorescence intensity was observed in the hydrogen peroxide-treated HaCaT cells following JC-1 dye staining, indicative of mitochondrial depolarization. However, pretreatment with luteolin significantly inhibited the loss of MMP in the hydrogen peroxide-treated cells. Altered cell morphology was also observed in response to hydrogen peroxide treatment, however, the cell morphology was maintained and the overall cell shape was maintained following treatment with increased dosed of luteolin, indicating the protective effect of luteolin against hydrogen peroxide-induced cell death.

Luteolin inhibits hydrogen peroxide-induced keratinocyte apoptosis through the PI3K/AKT pathway. To assess the mechanism by which luteolin treatment can protect against hydrogen peroxide-induced keratinocyte apoptosis, the present study investigated whether luteolin pretreatment was associated with varied intracellular signaling pathway activation. As shown in Fig. 3, treatment of the keratinocytes with hydrogen peroxide resulted in a significant inhibition of PI3K/AKT pathway activation, which indicated the inhibition of cell growth and differentiation. Consistent with this observation, there was increased expression of BAX and decreased expression of BCL-2, and the BCL-2/BAX ratio were decreased, which suggested that these cells underwent apoptosis once exposed to hydrogen peroxide treatment.

Luteolin pretreatment not only significantly restored the cell viability, but also decreased the apoptotic rate, upregulated the expression of BCL-2, downregulated the expression of $\mathrm{BAX}$ and increased the BCL-2/BAX ratio. In addition, luteolin pretreatment increased the phosphorylation of AKT in a dose-dependent manner, as the PI3K/AKT pathway is one of the most important intracellular survival signaling pathways. This result suggested the protective effect of luteolin in I/R injury may be associated with PI3K/AKT pathway activation.

Luteolin treatment protects against skin damage during the process of $I / R$. From the observations in vitro, it was hypothesized that luteolin may have a protective effect in I/R injury during skin flap surgery. To evaluate this hypothesis, the present study successfully established a cutaneous I/R injury rat model. To assess the effect of luteolin on I/R injury in the skin flap model, the surviving areas of the flaps were measured 7 days following surgery. It was observed that the I/R injury group exhibited smaller surviving flap areas compared with the mock control groups. The rats that received luteolin pretreatment had larger surviving flap areas than those in the I/R injury group at 7 days following surgery (Fig. 4A).

It was also observed that luteolin treatment suppressed the mRNA levels of pro-inflammatory cytokines and chemokines. The expression of pro-inflammatory cytokines in the biopsied skin samples were examined using an RT-qPCR assay. As shown in Fig. 4B, the expression levels of TNF- $\alpha$, IL-6 and IL-1 $\beta$ were significantly decreased in the luteolin-treated 

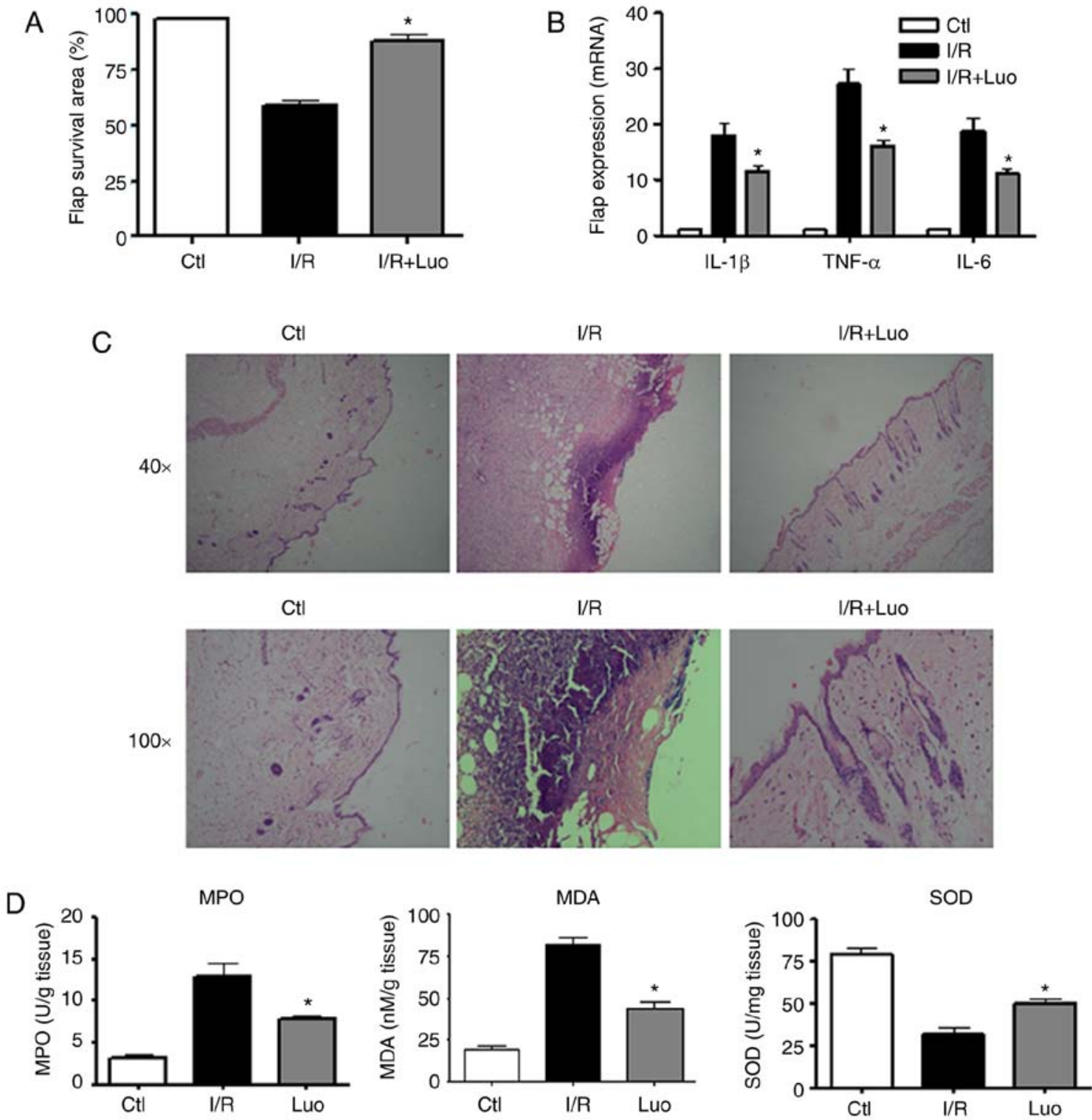

Figure 4. Luteolin administrations ameliorates I/R injury-induced skin tissue damage by inhibition of acute inflammation and oxidative stress levels. (A) Pro-survival effects of luteolin on I/R-injured skin tissue in rats undergoing skin flap surgery; flap survival area was significantly improved in rats subjected to I/R injury with luteolin treatment. (B) Relative expression of pro-inflammatory cytokines in the skin tissue biopsy was detected by reverse transcription-quantitative polymerase chain reaction analysis. (C) I/R-injured skin tissues were stained with hematoxylin and eosin and the histopathologic changes were visualized under a light microscope at different magnifications (x40 and x100). (D) Effect of luteolin administration on I/R-induced oxidative damage in skin tissue; the relevant biomarkers were assessed using commercial kits. Values are expressed as the mean \pm standard deviation $(\mathrm{n}=8)$. ${ }^{*} \mathrm{P}<0.05$, vs. Ctl. I/R, ischemia-reperfusion; Luo, luteolin; IL, interleukin; TNF- $\alpha$, tumor necrosis factor; Ctl, control; MPO, myeloperoxidase; MDA, malondialdehyde; SOD, superoxide dismutase.

groups compared with those in the I/R injury groups. The histopathological examination indicated that the severity of tissue injury in the luteolin pretreatment groups was markedly reduced; there was less inflammatory cell infiltration and the damaged skin area was significantly decreased (Fig. 4C).

To assess the I/R injury induced oxidative stress damage, the levels of MPO, MDA, and SOD were examined in the surgical flaps. There was increased production of MPO and MDA in the ischemia group compared with the control group, and luteolin treatment significantly decreased the expression of these enzymes. The decreased expression of SOD following $\mathrm{I} / \mathrm{R}$ injury was recovered in response to luteolin treatment. These results indicated the oxidative stress scavenging effects of luteolin (Fig. 4D).
Protective effect of luteolin on $I / R$-induced skin damage is partly mediated through activation of the PI3K/AKT pathway. Using immunofluorescent staining (Fig. 5A and B), it was observed that there was increased activation of caspase- 3 protein in the ischemia group compared with the control group and the luteolin treatment group. Luteolin treatment reduced the level of cell apoptosis induced by I/R injury, as the protein expression of activated caspase-3 was significantly reduced. The levels of phospho-AKT and antioxidant enzyme HO-1 were significantly increase in response to luteolin treatment, indicating that these two molecules may be involved in the protective function of luteolin.

To determine whether luteolin-induced skin protection was mediated by the PI3K/AKT pathway, the PI3K inhibitor 
A

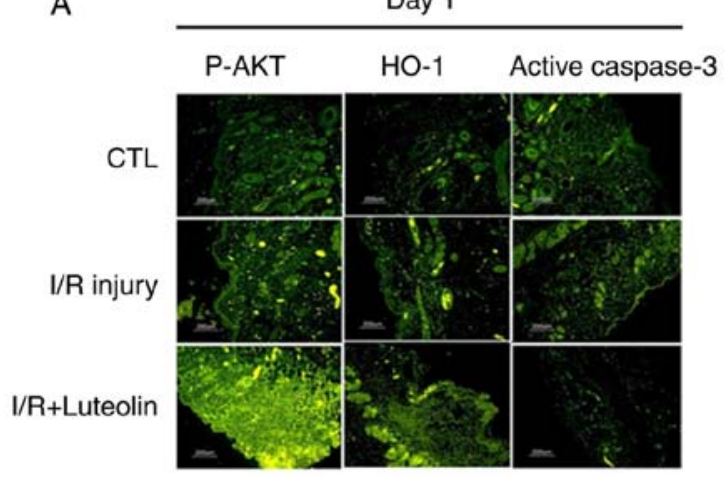

B
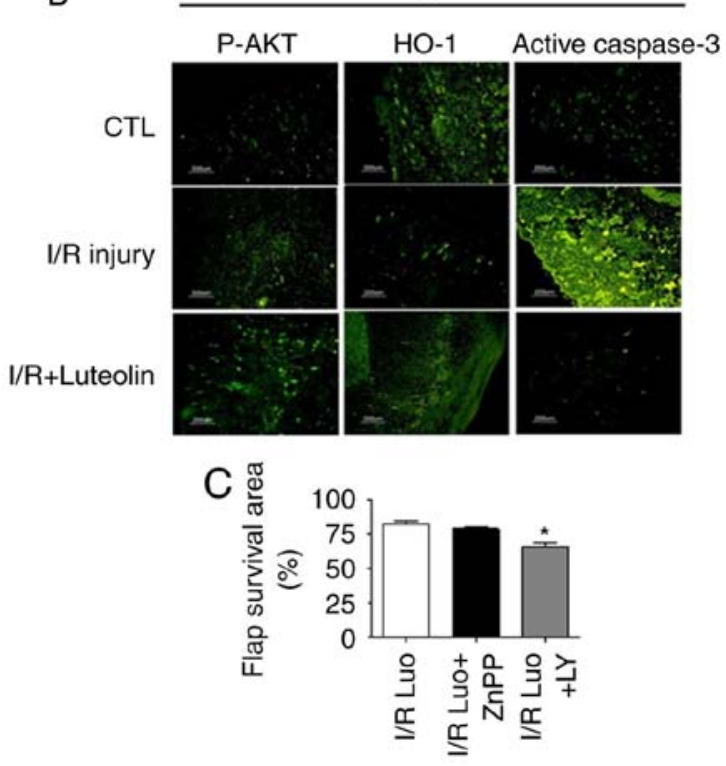

Figure 5. Protective effect of luteolin against I/R injury is mediated via the PI3K/AKT pathway. Immunofluresence staining of the skin tissue sections isolated (A) 1 day and (B) 7 days post-I/R injury. (C) Flap survival area in rats subjected to $\mathrm{I} / \mathrm{R}$ injury and luteolin treatment were reduced in the rats that received intraperitoneal injection of PI3K/AKT inhibitor LY294002, but not by HO-1 inhibitor ZnPP. "P<0.05, vs. Ctl. I/R, ischemia-reperfusion; PI3K, phosphoinositide-3-kinase; AKT, protein kinase B; HO-1, heme oxygenase-1; Luo, luteolin; LY, LY294002; CTL, control.

LY294002 (14) was used. The inhibition of AKT activity markedly reduced the luteolin-induced protection and inhibition of apoptosis in skin I/R injury (Fig. 5C). These results suggested that luteolin inhibited apoptosis and improved skin flap survival at least partly through the PI3K/AKT pathway in skin flap surgery. By contrast, using the HO-1 inhibitor $\mathrm{ZnPP}$ (15), no significant change in luteolin-induced protection was observed; the surviving flap areas and neutrophil infiltration levels were similar to those in the luteolin treatment groups, suggesting that HO-1 may not be a crucial molecule in regulation of skin damage during the I/R injury caused by skin flap surgery.

\section{Discussion}

During skin flap surgery, the skin tissue is lifted from a donor site and moved to a recipient site with an intact blood supply, and $I / R$ injury is the main factor reducing the survival rate of flaps following grafting (15). I/R injury refers to the tissue and microvasculature injury that is observed despite the restoration of blood flow following an initial ischemic insult, often affecting free flaps. The mechanisms of I/R injury include hypoxia, inflammation and oxidative damage, characterized by microvascular vasoconstriction, ROS release, oxidant/antioxidant imbalance and neutrophil adhesion/infiltration (16).

Flavonoids are a group of natural products currently receiving attention for their anti-reactive stress and anti-inflammatory effects. Several studies have reported that certain flavonoids, including kaempferol and quercetin, exert antioxidant effects and can also inhibit tissue damage (17). Luteolin is a common flavonoid that exists in several types of plants including fruits, vegetables, and medicinal herbs; it has antioxidant, anti-inflammatory, antitumor or other physiological beneficial effects. Studies have demonstrated that luteolin may exert protective effects in I/R injury in various pathological conditions, including myocardial and cerebral I/R injury $(18,19)$. In our previous study, it was shown that luteolin protected HUVECs from TNF- $\alpha$-induced oxidative stress and inflammation (20). In the present study, an in vivo animal model was used to investigate the protective effect of luteolin against cutaneous I/R injury during the process of skin flap surgery. It was found that luteolin pretreatment conferred a skin protective effect, as evidenced by improvement following I/R injury and reduced skin keratinocyte apoptosis. Of note, PI3K/AKT signaling was shown to be key in this process. The findings indicate that luteolin protected against skin tissue damage in rats that underwent skin flap surgery.

There are diverse mechanisms of cell death, including necrosis, apoptosis, mitotic catastrophe and pyroptosis (21). Apoptosis is an active form of cell death that is initiated by a variety of stimuli, including ROS (22). Several studies have established that I/R injury can induce cell apoptosis, resulting in the deregulation of associated functions (23-25). ROS-induced cell apoptosis has been shown to be one of the important pathological features of cutaneous I/R injury.

To understand the cytotoxic protective effect of luteolin in I/R injury, the present study first measure the protective effect of luteolin using the illustrative human keratinocyte $\mathrm{HaCaT}$ cell line as an in vitro skin model, as skin keratinocytes are the predominant cell type in the epidermis, constituting $90 \%$ of the cells found in the outermost layer of the skin (26). During skin I/R injury, ROS-induced skin keratinocyte apoptosis has been considered to be the major pathological cause for the tissue damage (27). In the present study, by analyzing the hydrogen peroxide-induced skin $\mathrm{HaCaT}$ cell apoptosis, the results showed that luteolin pretreatment significantly inhibited the hydrogen peroxide-induced apoptosis, indicating the anti-apoptotic property of luteolin.

To further delineate the mechanism, the present study also measured the expression of apoptosis regulatory components. Apoptosis is mediated by two evolutionarily conserved pathways: Intrinsic and extrinsic cell death pathways, which are respectively represented by the Bcl-2 family and the caspase family. The Bcl-2 family proteins, consisting of death antagonists (Bcl-2) and agonists (Bax), are crucial in the regulation of ROS-induced cell death (28). It has been found that, during ischemia and particularly when combined with reperfusion, Bax protein is triggered and translocated into the outer 
mitochondrial membrane, resulting in elevated Bax levels and a reduced $\mathrm{Bcl}-2 / \mathrm{Bax}$ ratio (29). It is well known that this ratio is involved in MMP. The downregulation of the $\mathrm{Bcl}-2 / \mathrm{Bax}$ ratio indicates that mitochondria-dependent pathways are involved in hydrogen peroxide-induced apoptosis (30). It has been shown that the overexpression of Bcl-2 decreases cell apoptosis in multiple types of I/R injury (31). In the present study, it was detected that luteolin pretreatment significantly elevated the expression of BCL-2 and decreased the expression of BAX, which corresponded to the increased BCL-2/BAX ratio. Therefore changes in the ratio of pro-apoptotic to anti-apoptotic proteins may contribute to the observed anti-apoptotic mode of action of luteolin.

Caspase- 3 are cysteine proteases are central in the execution of the apoptotic program. Caspase-3 interacts with caspase- 8 and caspase- 9 , therefore, caspase- 3 is activated in the apoptotic cell by extrinsic (death ligand) and intrinsic (mitochondrial) pathways (32). In the present study, marked Caspase-3 activation was observed in the healing skin tissue following skin flap surgery, indicating that ROS-induced apoptosis contributed to the I/R injury-induced tissue damage, The anti-apoptotic action of luteolin alleviated the tissue damage during the cutaneous I/R injury, and the in vitro experiments support this conclusion.

Cutaneous I/R injuries also cause the development of inflammatory responses (33). In the present study, the increased expression of pro-inflammatory cytokines IL-1 $\beta$ and TNF- $\alpha$ suggested the induced acute inflammation upon cutaneous I/R injury. It was noted that luteolin treatment attenuated the acute inflammation by decreasing the expression of these pro-inflammatory cytokines, suggesting that the anti-inflammatory properties of luteolin may accelerate the wound healing process. Neutrophil infiltration is characteristic of acute inflammation, which has been suggested to aggravate the reperfusion injury induced by leukocyte activation, and the expression of adhesion molecules contributed by ROS. In the present study, the increased expression of MPO, MDA and inflammatory factors in the injured skin tissue during cutaneous I/R injury were significantly ameliorated, and the decreased release of ROS and increased production of antioxidant enzymes SOD and HO-1 upon luteolin treatment support the anti-inflammatory and ROS scavenging function of luteolin.

The PI3K/AKT pathway is one of the well-documented pathways involved in protection against oxidative stress and is critical in promoting wound healing (34). The activation of PI3K/AKT has been shown to have a beneficial effect on multiple types of I/R injury, including the gut, liver, heart and cerebral regions (35-39). The phosphorylation of AKT has been shown to suppress apoptosis and promote cell survival in I/R injury (40). AKT regulates cell survival by phosphorylating different substrates that directly or indirectly regulate apoptosis. It has been found that the phosphorylation of AKT prevents cytochrome $c$ release by inhibiting the interaction of BCL-2 family apoptotic proteins, including BCL-extra large, with other apoptosis regulating molecules, including BCL-2-associated death promoter; AKT also phosphorylates caspase-9 on Ser196, which inhibits its proteolytic activity via a conformation change (41). Previous studies have demonstrated that activation of the PI3K/AKT signaling pathway improved wound healing in human and animal models; the increased $\mathrm{PI} 3 \mathrm{~K} / \mathrm{AKT}$ activation during the wound healing process was time course-dependent, and was mainly observed in the early period during wound healing $(21,42)$. In the present study, it was found that luteolin pretreatment significantly upregulated the protein phosphorylation of PI3K and AKT. The increased activation of the PI3K/AKT signaling pathway in the luteolin treatment group, which occurred mainly on Day 1 rather than Day 7, suggested that activation of the PI3K/AKT pathway was an early event for tissue regeneration. Therefore, the protective effect of luteolin was due, at least in part, to its ability to upregulate the activation of the PI3K/AKT signaling pathway.

To understand whether the protective effects of luteolin were mediated via the PI3K/AKT pathway, the rats received pretreatment with PI3K/AKT inhibitors during the in vivo administration of luteolin. The results showed that, in the presence of LY294002, the cytoprotective activity of luteolin was significantly reduced, suggesting the involvement of the PI3K/AKT pathway in the regulation of cutaneous $\mathrm{I} / \mathrm{R}$ injury.

In conclusion, the present study demonstrated that luteolin pretreatment attenuated cutaneous I/R injury by scavenging of extracellular ROS and regulating apoptosis. Therefore, the administration of luteolin may represent a promising therapeutic strategy for the treatment of ROS-related cutaneous I/R injury and improve skin flap survival.

\section{Acknowledgements}

Not applicable.

\section{Funding}

This study was supported by a grant from the Natural Science Foundation of Jiangsu Province China (grant nos. BK20141505 and BK20171347), the Key Laboratory of Acupuncture and Medicine Research (grant no. 201710zykf01), Qinglan Project of Jiangsu Province and the Research Projects in Traditional Chinese Medicine Industry of China (grant no. 201507004-2).

\section{Availability of data and materials}

The datasets used and/or analyzed during the current study are available from the corresponding author on reasonable request.

\section{Authors' contributions}

HW and YS contributed to planning and implementing the experiments, interpretation of data and writing the manuscript. GC, HS and LZ contributed to implementing the experiments. $\mathrm{ZS}$ and $\mathrm{JH}$ contributed to planning the experiments, interpreting data, and writing the manuscript. All authors reviewed the manuscript.

\section{Ethics approval and consent to participate}

All animal experiments were approved by the Institutional Animal Care and Use Committee of Nanjing University (Nanjing, China). 


\section{Patient consent for publication}

Not applicable.

\section{Competing interests}

The authors declare that they have no competing interests.

\section{References}

1. Wang X, Takahashi N, Uramoto H and Okada Y: Chloride channel inhibition prevents ROS-dependent apoptosis induced by ischemia-reperfusion in mouse cardiomyocytes. Cell Physiol Biochem 16: 147-154, 2005.

2. Park TH and Park YJ: The effect of botulinum toxin a on ischemia-reperfusion injury in a rat model. Biomed Res Int 2017: 1074178, 2017.

3. Nabavi SF, Braidy N, Gortzi O, Sobarzo-Sanchez E, Daglia M, Skalicka-Woźniak K and Nabavi SM: Luteolin as an anti-inflammatory and neuroprotective agent: A brief review. Brain Res Bull 119: 1-11,2015.

4. Lee JP, Li YC, Chen HY, Lin RH, Huang SS, Chen HL, Kuan PC, Liao MF, Chen CJ and Kuan YH: Protective effects of luteolin against lipopolysaccharide-induced acute lung injury involves inhibition of MEK/ERK and PI3K/Akt pathways in neutrophils. Acta Pharmacol Sin 31: 831-838, 2010.

5. Nunes C, Almeida L, Barbosa RM and Laranjinha J: Luteolin suppresses the JAK/STAT pathway in a cellular model of intestinal inflammation. Food Funct 8: 387-396, 2017.

6. Das M, Ram A and Ghosh B: Luteolin alleviates bronchoconstriction and airway hyperreactivity in ovalbumin sensitized mice. Inflamm Res 52: 101-106, 2003.

7. Shanmugam S, Thangaraj P, Lima B, Lima BDS, Chandran R, de Souza Araújo AA, Narain N, Serafini MR and Júnior LJQ: Effects of luteolin and quercetin 3- $\beta$-d-glucoside identified from Passiflora subpeltata leaves against acetaminophen induced hepatotoxicity in rats. Biomed Pharmacother 83: 1278-1285, 2016.

8. Hong X, Zhao X, Wang G, Zhang Z, Pei H and Liu Z: Luteolin treatment protects against renal ischemia-reperfusion injury in rats. Mediators Inflamm 2017: 9783893, 2017.

9. Yu D, Li M, Tian Y, Liu J and Shang J: Luteolin inhibits ROS-activated MAPK pathway in myocardial ischemia/reperfusion injury. Life Sci 122: 15-25, 2015.

10. Karakas BR, Davran F, Elpek GO, Akbas SH, Gulkesen KH and Bulbuller N: The effects of luteolin on the intestinal ischemia/reperfusion injury in mice. J Invest Surg 27: 249-255, 2014.

11. Gideroglu K, Yilmaz F, Aksoy F, Bugdayci G, Saglam I and Yimaz F: Montelukast protects axial pattern rat skin flaps against ischemia/reperfusion injury. J Surg Res 157: 181-186, 2009.

12. Kim HJ, Xu L, Chang KC, Shin SC, Chung JI, Kang D, Kim SH, Hur JA, Choi TH, Kim S and Choi J: Anti-inflammatory effects of anthocyanins from black soybean seed coat on the keratinocytes and ischemia-reperfusion injury in rat skin flaps. Microsurgery 32: 563-570, 2012.

13. Yang Y, Zhang Y, Wang Z, Wang S, Gao M, Xu R, Liang C and Zhang $\mathrm{H}$ : Attenuation of acute phase injury in rat intracranial hemorrhage by cerebrolysin that inhibits brain edema and inflammatory response. Neurochem Res 41: 748-757, 2016.

14. Yoon JJ, Jeong JW, Choi EO, Kim MJ, Hwang-Bo H, Kim HJ, Hong SH, Park C, Lee DH and Choi YH: Protective effects of Scutellaria baicalensis Georgi against hydrogen peroxide-induced DNA damage and apoptosis in $\mathrm{HaCaT}$ human skin keratinocytes. EXCLI J 16: 426-438, 2017.

15. Birk-Sorensen L, Kerrigan CL and Jensen GS: E-selectin and $\mathrm{L}$-selectin blockade in pure skin flaps exposed to ischaemia and reperfusion injury. Scand J Plast Reconstr Surg Hand Surg 32: 365-371, 1998.

16. Francis A and Baynosa RC: Hyperbaric oxygen therapy for the compromised graft or flap. Adv Wound Care (New Rochelle) 6 : 23-32, 2017.

17. Kumar AD, Bevara GB, Kaja LK, Badana AK and Malla RR: Protective effect of 3-O-methyl quercetin and kaempferol from semecarpus anacardium against $\mathrm{H} 2 \mathrm{O} 2$ induced cytotoxicity in lung and liver cells. BMC Complement Altern Med 16: 376 , 2016 .
18. Zhang X, Du Q, Yang Y, Wang J, Dou S, Liu C and Duan J: The protective effect of Luteolin on myocardial ischemia/reperfusion (I/R) injury through TLR4/NF- $\mathrm{KB} / \mathrm{NLRP} 3$ inflammasome pathway. Biomed Pharmacother 91: 1042-1052, 2017.

19. Zhang S, Qi Y, Xu Y, Han X, Peng J, Liu K and Sun CK: Protective effect of flavonoid-rich extract from rosa laevigata michx on cerebral ischemia-reperfusion injury through suppression of apoptosis and inflammation. Neurochem Int 63: 522-532, 2013.

20. Sun GB, Sun X, Wang M, Ye JX, Si JY, Xu HB, Meng XB, Qin M, Sun J, Wang HW and Sun XB: Oxidative stress suppression by luteolin-induced heme oxygenase-1 expression. Toxicol Appl Pharmacol 265: 229-240, 2012.

21. Kroemer G, Galluzzi L, Vandenabeele P, Abrams J, Alnemri ES, Baehrecke EH, Blagosklonny MV, El-Deiry WS, Golstein P, Green DR, et al: Classification of cell death: Recommendations of the nomenclature committee on cell death 2009. Cell Death Differ 16: 3-11, 2009.

22. Miyata Y, Matsuo T, Sagara Y, Ohba K, Ohyama K and Sakai H: A mini-review of reactive oxygen species in urological cancer: Correlation with NADPH oxidases, angiogenesis, and apoptosis. Int J Mol Sci 18: E2214, 2017

23. Liu H, Jing X, Dong A, Bai B and Wang H: Overexpression of TIMP3 protects against cardiac ischemia/reperfusion injury by inhibiting myocardial apoptosis through ROS/mapks pathway. Cell Physiol Biochem 44: 1011-1023, 2017.

24. Uchiyama A, Yamada K, Perera B, Ogino S, Yokoyama Y, Takeuchi Y, Ishikawa $\mathrm{O}$ and Motegi S: Topical betamethasone butyrate propionate exacerbates pressure ulcers after cutaneous ischemia-reperfusion injury. Exp Dermatol 25: 678-683, 2016

25. Uchiyama A, Yamada K, Perera B, Ogino S, Yokoyama Y, Takeuchi Y, Ishikawa O and Motegi SI: Protective effect of MFG-E8 after cutaneous ischemia-reperfusion injury. J Invest Dermatol 135: 1157-1165, 2015.

26. Johansen C: Generation and culturing of primary human keratinocytes from adult skin. J Vis Exp 130: e56863, 2017.

27. Cai Y, Wang W, Liang H, Sun L, Teitelbaum DH and Yang H: Keratinocyte growth factor improves epithelial structure and function in a mouse model of intestinal ischemia/reperfusion. PLoS One 7: e44772, 2012.

28. Zeng C, Jiang W, Zheng R, He C, Li J and Xing J: Cardioprotection of tilianin ameliorates myocardial ischemia-reperfusion injury: Role of the apoptotic signaling pathway. PLoS One 13: e193845, 2018.

29. Xiao YD, Liu YQ, Li JL, Ma XM, Wang YB, Liu YF, Zhang MZ, Zhao PX, Xie F and Deng ZX: Hyperbaric oxygen preconditioning inhibits skin flap apoptosis in a rat ischemia-reperfusion model. J Surg Res 199: 732-739, 2015.

30. Roy AM,Baliga MS and KatiyarSK: Epigallocatechin-3-gallate induces apoptosis in estrogen receptor-negative human breast carcinoma cells via modulation in protein expression of p53 and Bax and caspase-3 activation. Mol Cancer Ther 4: 81-90, 2005.

31. Liu YQ, Liu YF, Ma XM, Xiao YD, Wang YB, Zhang MZ, Cheng AX, Wang TT, Li JL, Zhao PX, et al: Hydrogen-rich saline attenuates skin ischemia/reperfusion induced apoptosis via regulating Bax/Bcl-2 ratio and ASK-1/JNK pathway. J Plast Reconstr Aesthet Surg 68: e147-e156, 2015.

32. Salvesen GS: Caspases: Opening the boxes and interpreting the arrows. Cell Death Differ 9: 3-5, 2002.

33. Ju J, Wu J and Hou R: Role of the p38 mitogen-activated protein kinase signaling pathway in estrogen-mediated protection following flap ischemia-reperfusion injury. Cell Biochem Funct 34: 522-530, 2016.

34. Zhang B, Zhao Z, Meng X, Chen H, Fu G and Xie K: Hydrogen ameliorates oxidative stress via PI3K-Akt signaling pathway in UVB-induced HaCaT cells. Int J Mol Med 41: 3653-3661, 2018.

35. Mahajan UB, Patil PD, Chandrayan G, Patil CR, Agrawal YO, Ojha S and Goyal SN: Eplerenone pretreatment protects the myocardium against ischaemia/reperfusion injury through the phosphatidylinositol 3-kinase/Akt-dependent pathway in diabetic rats. Mol Cell Biochem 446: 91-103, 2018.

36. Jiao $\mathrm{S}$, Zhu H, He $\mathrm{P}$ and Teng J: Betulinic acid protects against cerebral ischemia/reperfusion injury by activating the PI3K/Akt signaling pathway. Biomed Pharmacother 84: 1533-1537, 2016.

37. Kai-lan W and Si Z: Pretreatment with erythropoietin attenuates intestinal ischemia reperfusion injury by further promoting PI3K/Akt signaling activation. Transplant Proc 47: 1639-1645, 2015. 
38. Arslan F, Lai RC, Smeets MB, Akeroyd L, Choo A, Aguor EN, Timmers L, van Rijen HV, Doevendans PA, Pasterkamp G, et al: Mesenchymal stem cell-derived exosomes increase ATP levels, decrease oxidative stress and activate PI3K/Akt pathway to enhance myocardial viability and prevent adverse remodeling after myocardial ischemia/reperfusion injury. Stem Cell Res 10: 301-312, 2013.

39. Mason S, Hader C, Marlier A, Moeckel G and Cantley LG: Met activation is required for early cytoprotection after ischemic kidney injury. J Am Soc Nephrol 25: 329-337, 2014.

40. Thokala S, Inapurapu S, Bodiga VL, Vemuri PK and Bodiga S: Loss of ErbB2-PI3K/Akt signaling prevents zinc pyrithione-induced cardioprotection during ischemia/reperfusion. Biomed Pharmacother 88: 309-324, 2017.
41. Nath S, Mandal C, Chatterjee U and Mandal C: Association of cytosolic sialidase Neu2 with plasma membrane enhances Fas-mediated apoptosis by impairing PI3K-Akt/mTOR-mediated pathway in pancreatic cancer cells. Cell Death Dis 9: 210, 2018.

42. Yu T, Gao M, Yang P, Pei Q, Liu D, Wang D, Zhang X and Liu Y: Topical insulin accelerates cutaneous wound healing in insulin-resistant diabetic rats. Am J Transl Res 9: 4682-4693, 2017.

This work is licensed under a Creative Commons Attribution-NonCommercial-NoDerivatives 4.0 International (CC BY-NC-ND 4.0) License. 